\title{
Influence of the coupling between an atmospheric pressure ion mobility spectrometer and the low pressure ion inlet of a mass spectrometer on the mobility measurement
}

\author{
Frank Gunzer \\ Physics Department, German University in Cairo, Cairo, Egypt
}

\begin{abstract}
Ion mobility spectrometers (IMS) are versatile gas analyzers. Due to their small size and robustness, combined with a very high sensitivity, they are often used in gas sensing applications such as environmental monitoring. In order to improve the selectivity, they are typically combined with a mass spectrometer (MS). Since IMS works at atmospheric pressure, and MS works at vacuum, a special interface reducing the pressure over normally two stages has to be used. In this paper the influence of this coupling of different pressure areas on the IMS signal will be analyzed with help of finite elements method simulations.
\end{abstract}

\section{Introduction}

Ion mobility spectrometry is a well known technique in order to analyze gases. The corresponding spectrometers are small, robust, sensitive and fast. Their selectivity, i.e. the capability of giving clearly different responses for different substances, is relatively limited [1]. Thus, they are often combined with mass spectrometers. Then not only the mobility of a substance can be determined, but also the molecular mass of the analytes. The amount of information that can be used for identification is increased [2].

IMS work by employing a time-of-flight principle. The analytes are ionized, and these ions are accelerated with help of electric fields. Then they are, again with help of electric fields, driven towards the detector, which is located at a certain distance from the ionization location. By measuring the time the ions need to travel that distance, their mobility can be determined. Since IMS devices do not use vacuum (one factor that makes their size small), the ions constantly collide with ambient air molecules on the way to the detector. Thus it is their mobility that decides how fast they can reach the detector while drifting towards it [1].

MS can work with many principles [3], but all these different principles have in common that analyte ions are accelerated with help of electric fields in vacuum. One version is e.g. the time-of-flight mass spectrometry, where the ions, similar to IMS, travel a certain distance to a detector. Since they travel in vacuum, it is not the mobility and the collisions with air molecules that determine their velocity and thus their travel time, but their kinetic energy provided by the electric acceleration field and thus their mass. The flight time allows for a direct calculation of the molecular mass of the analytes.
Combining IMS and MS is relatively straight forward, since they both need analyte ions to obtain the corresponding information. One major obstacle is, however, the different pressure regimes. The ions have to be brought from an area of atmospheric pressure (1000 mbar) to the vacuum of the MS (ca. $5 \times 10^{-7}$ mbar). This if often done via two stages, where an intermediate stage has a pressure of a few mbar.

The problem is that the opening at the interface of the IMS to the first pressure reduction stage changes the air flow in the IMS. Often, the IMS itself is then operated at reduced pressure. But then the ion chemistry is changed. This might be no problem if just the identification of analytes is to be performed, but might be problematic if further information is to be obtained that relies on the ion chemistry inside the IMS (e.g. clustering or dimer formation). Thus, atmospheric pressure IMS/MS combinations are very interesting and important, with the before-mentioned problems regarding the changed air flow in the IMS.

In this paper we have analyzed with help of finite elements method (FEM) simulations the change of the ion motion through the IMS when directly combined with an intermediate vacuum stage of a few mbar pressure. After a short description of the set up, the paper will show how the IMS response is directly affected by the pressure change caused by the interface to the reduced pressure stage.

\section{Experimental}

Figure 1 shows the typical set up of commercial IMS devices used in our laboratory [4-8]. On the left is region where the ions are formed. 


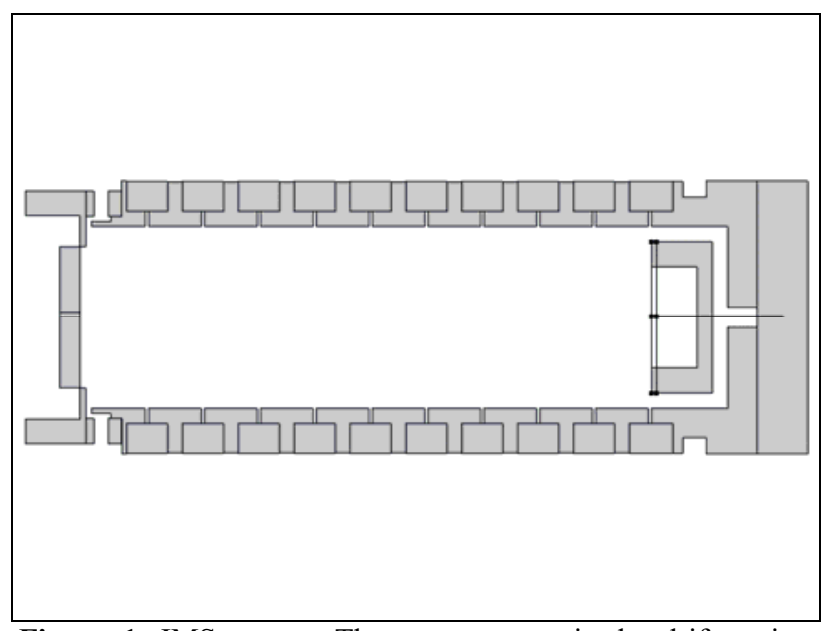

Figure 1. IMS set up. The center space is the drift region, enclosed on top and bottom by $\mathrm{T}$-shaped electrodes. The detector is located on the right at the end of the drift region (here shown by two parallel, vertical lines at very small distance). In the middle, starting at the detector is the small tunnel leading into the low pressure area which is the last rectangle on the far right.

The largest part is the drift tube, where a constant electric field lets the ions drift towards the detector. In this drift region, the ions are separated according to their mobility, which allows them to reach different velocities when colliding with the ambient air molecules and thus to reach the detector after substance-specific drift times.

The connection into the low pressure stage can be realized by different means. One successful approach is to use a small diameter tunnel, where the diameter is ca. $200 \mu \mathrm{m}$ (length $2 \mathrm{~cm}$ ) so that the high pressure can be maintained in the drift tube. The whole length is ca. 5.5 $\mathrm{cm}$ from left to right; the drift region is $5 \mathrm{~cm}$ wide (at a diameter of $2 \mathrm{~cm}$ ). Inside the drift region, a low air current against the direction of the ions is supporting the separation process. This air current start on the right above and below the detector, where openings of ca. 1 $\mathrm{mm}$ diameter allow a flow of $200 \mathrm{ml} / \mathrm{min}$ to enter the drift region; the outlet is located on the left above and below the space where the ions are created.

The electrodes are supplied with a voltage difference of $2000 \mathrm{~V}$ between the entrance into the drift region and the detector, where a corresponding voltage divider leads to a uniform voltage drop from electrode to electrode when going from left to right. Thus a uniform electric field can be obtained at the centre of the drift region.

The ions are created in a small rectangular space in the middle of the region on the left of the drift region (diameter ca. $0.5 \mathrm{~mm}$ ). This is the typical size when using an electron gun as we normally do in our experiments. The concentration is quite low with $10^{-13} \mathrm{~mol} / \mathrm{m}^{3}$ in order to be able to ignore Coulomb repulsion.

The simulation has been carried out by calculating the electric field with help of Maxwell's Equations. Ion-ionrepulsion in form of Coulomb Repulsion has, as said before, been ignored. The air currents have been simulated by solving typical computational fluid dynamics (CFD) equations for the k- $\varepsilon$-turbulence model [9]. The pressure at the outlets on the left were $10^{5} \mathrm{~Pa}$, the low pressure area was set to different pressures between
$7.5 \times 10^{4} \mathrm{~Pa}$ and $10^{2} \mathrm{~Pa}$. The ions' motion has been calculated by solving the Navier-Stokes-Equations under the influence of the electric field and the convection flow field (i.e., the fields calculated in a previous step with help of Maxwell's Equations and CFD). The mesh size has been set to max element sizes of $0.05 \mathrm{~mm}$, the mesh itself was created by the software (COMSOL Multiphysics, v. 4.3b, www.comsol.com).

\section{Results and Discussion}

The initial simulation used pressure values as they are typical in such a set up. On the outlet of the IMS $10^{5} \mathrm{~Pa}$ pressure (outlet leads into ambient atmosphere), the low pressure side $400 \mathrm{~Pa}$ (typical pressure in reduced pressure stage), inlet into IMS provides $200 \mathrm{ml} / \mathrm{min}$ air. Figure 2 shows the air current calculated for that situation near the spot where the ions are created.

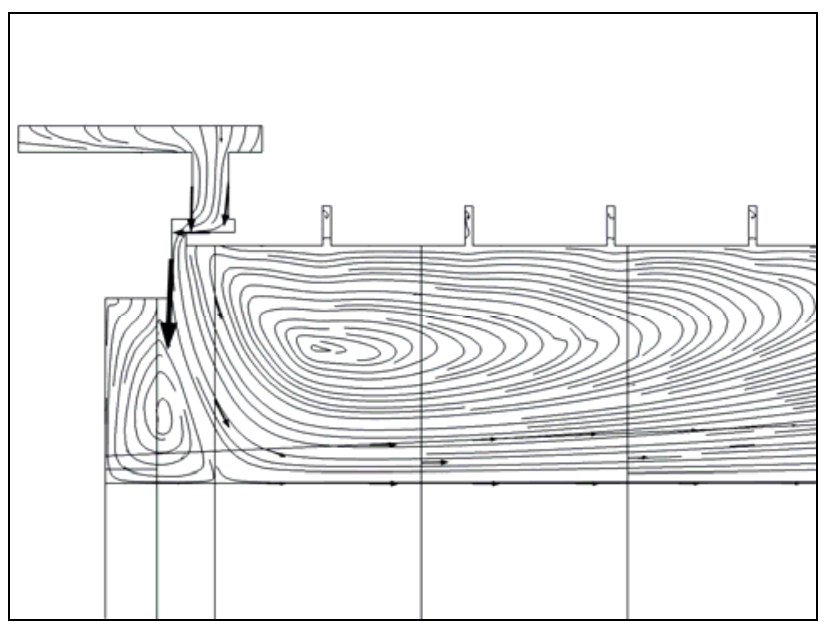

Figure 2. Calculated air current at the beginning of the drift region, only upper half of the device shown. The outlet at the top left of the drawing actually turns into an inlet (see arrow direction downward), and at the center of the device a current towards the right (i.e. the detector and the tunnel into the low pressure stage at $400 \mathrm{~Pa}$ pressure) forms.

As can be seen, the pressure difference is strong, so that at the outlet into ambient atmosphere actually the air is pushed into the device and not leaving it. At the center of the device, a current towards the right and thus towards the detector and the tunnel into the low pressure stage is created. Since the ions are created in our experiments near the center axis of the devices, the large turbulence in the drift region can actually be ignored; for standard set ups creating ion distributions over the whole width of the device this turbulence would be, however, problematic. The current in the middle is quite fast (much faster than the drift velocity of the ions, which is here typically 700 $\mathrm{cm} / \mathrm{s}$ ), and thus leads quickly to a deformation of the cloud. Figure 3 shows this deformation.

It becomes clear that the air current deforms the cloud and changes the effective velocity in comparison with an undisturbed cloud. The top picture in that figure shows a cloud a few hundred $\mu$ s after leaving the region of its 
creation (vertical black line as spatial reference). The rectangular structure is still dominant, which resembles the shape of the ionization region. The deformation by diffusion is at this moment not very strong. The bottom picture in that figure shows the deformation together with arrows indicating the direction of the air current. The motion of the cloud is slower (the cloud is after the same time only on the black reference line), and the shape is clearly not rectangular.

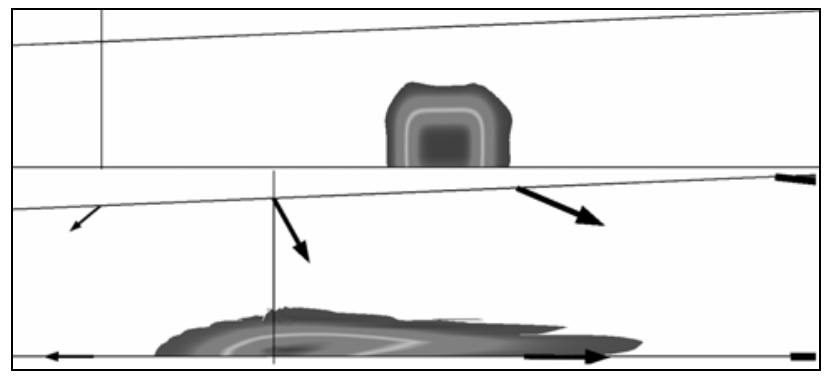

Figure 3. Top: undisturbed ion cloud. Bottom: Same cloud after the same travel time but this time disturbed by the air current. The cloud is closer to the reference line and does not show the same rectangular shape. Arrows show the direction of the air current. Due to the air flow against the motion of the ions induced by the electric field, the propagation into the direction of the detector is initially slower, but then faster.

The flow in the tunnel reaches a velocity of ca. $350 \mathrm{~m} / \mathrm{s}$ due to the pressure difference. Up to a pressure of $10^{3} \mathrm{~Pa}$ this situation does not change since the pressure difference is basically the pressure in the IMS; the contribution of the low pressure area is just too small (1 $\%)$. Thus, at these pressure differences the flow through the IMS is the same. Figure 4 shows the flow though the whole IMS in form of a velocity profile.

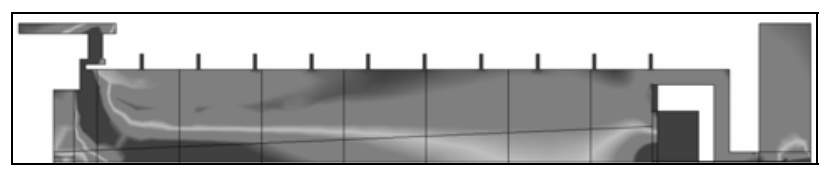

Figure 4. Velocity profile of the flow. Dark areas mean $10 \mathrm{~m} / \mathrm{s}$ and more. In the tunnel, the velocity reaches $350 \mathrm{~m} / \mathrm{s}$.

Due to the small dimensions of the cloud, there is still a good time signal to be obtained after the tunnel, i.e. the influence of the disturbance on the final signal form is tolerable. The problem is that due to the fast travel time, the function of the IMS is reduced even if the obtained signal is still of good quality. The travel time is only a fraction of the undisturbed case, and thus different analytes would have less time to separate. The resolving power of the device would thus be drastically reduced. In order to see how much the effect is, we have tested the deformation of the obtained of the obtained signal at different pressure differences.

As indicated before, the situation only changes if the pressure difference between the two areas is not dominated by the high pressure area alone. Thus, if the pressure in the low-pressure stage becomes comparable to the one in the IMS, the flow through the tunnel becomes less fast and thus the disturbance created in the IMS should be lower.

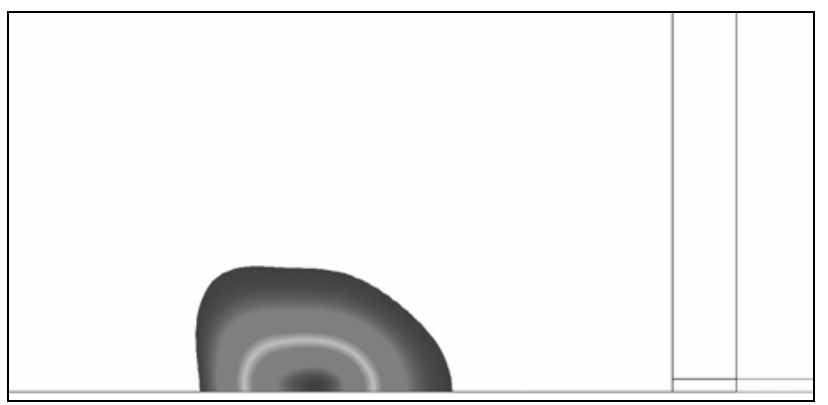

Figure 5. Ion cloud at the end of the drift region, pressure in low pressure stage is $7.5 \times 10^{4} \mathrm{~Pa}$.

Interestingly, only at a pressure of $7.5 \times 10^{4} \mathrm{~Pa}$ is the low pressure area, the distortion becomes negligible. The influence on the flight time is, however, not. Figure 5 shows the ion cloud shortly before the detector in this case. The cloud has a nearly symmetric shape. Actually, the cloud remains largely undeformed in hat situation, and only in the last $10 \mathrm{~mm}$ of the drift region the deformation sets in. The tunnel is then near enough so that even this relatively low pressure difference shows an effect due to the increase flow velocity in direction of the detector. The remaining area is almost unaffected, the outflow regions near the region where the ions enter the drift region is really an outlet and not an inlet.

Despite the small influence on the shape of the ion cloud, there is still a relevant influence on the flight time. Figure 6 shows the signal that the detector would measure. It has been obtained by integrating the concentration over the detector line.

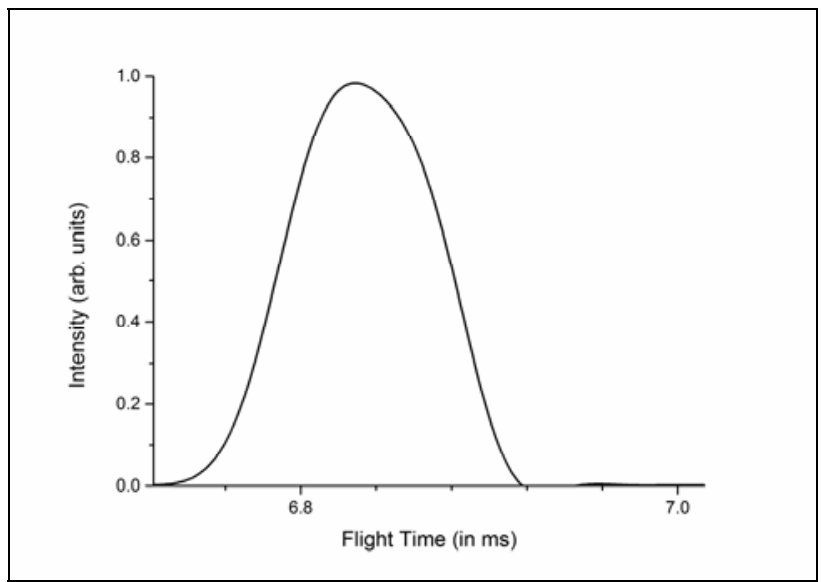

Figure 6. Ion cloud at the end of the drift region, pressure in low pressure stage is $7.5 \times 10^{4} \mathrm{~Pa}$.

The signal looks basically normal, but the high intensity areas are slightly distorted. Here it shows already that the velocity field is non-uniform and thus this asymmetry appears in the signal. This is again a comparably small distortion, much larger becomes the distortion of the total flight time which is demonstrated in figure 7. 


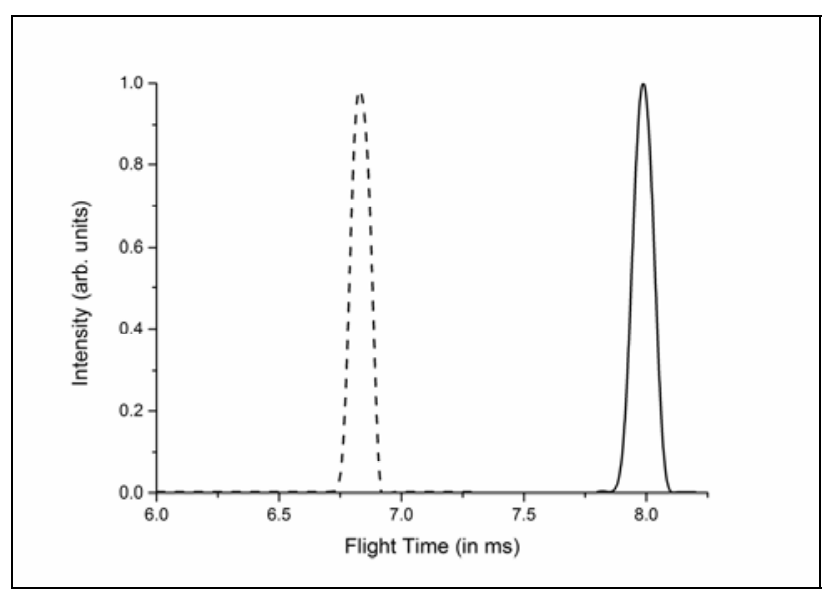

Figure 7. Ion cloud at the end of the drift region, pressure in low pressure stage is $7.5 \times 10^{4} \mathrm{~Pa}$.

The flight time is reduced by $15 \%$ from ca. $7.9 \mathrm{~ms}$ to ca. $6.8 \mathrm{~ms}$. The width (FWHM), however, is changing similarly from $97 \mu$ s to $87 \mu$ s so that the resolving power (which is in IMS the ratio of the signal width to the flight time) is nearly unchanged (based on a Gauss-Peak fit, which is in the case of the disturbed signal slightly less accurate compared with the undisturbed peak, where it is very accurate). The change in resolving power will be stronger with shorter flight times when using the lower pressures on the other side of the tunnel, and similarly the distortions. In facts, when the pressure difference is very large, the ion cloud concentrates on the center axis of the device so that an ion mobility measurement might even become impossible since no ions collide with the detector but enter the tunnel instead. In the case shown before, with only a relatively small pressure drop of 250 mbar, the number of ions hitting the detector and not entering the tunnel is only a third of the total ion number, i.e. two thirds enter the tunnel.

\section{Conclusions}

In this paper we have shown the influence of a low pressure area connected to an atmospheric pressure IMS by a tunnel with $100 \mu \mathrm{m}$ radius on the ion motion. Even at moderate pressure drops the ion cloud shows deformations with initially small changes of the resolving power. At a pressure reduction to $10^{4} \mathrm{~Pa}$ the effects are already strong, and the motion of the ions is largely dominated by the air current in the IMS. The velocity of this current exceeds then the velocity caused by the electric field and drift through the IMS by far. Thus there are negative effects on the resolving power which is proportional to the drift time. Furthermore, the number of ions hitting the detector at the end of the drift tube would be very low compared to the number of ions entering the tunnel. Thus a combined IMS/MS measurement as targeted by such a set up would not work. The results have been obtained for small ion clouds as they are typically created by an electron used in out experimental set up. Using other ionization principles yielding larger ion clouds might then be beneficial. However, the distortions would be quite large, too, since the air current formed by larger pressure drop in the drift region is quite irregular, as has been shown in this publication. It becomes obvious that the use of ion optics into the low pressure region is extremely helpful, possibly absolutely necessary if the low pressure stage is to be operated at the typical $400 \mathrm{~Pa}$ pressure.

In order to verify the validity of these simulations, experiments are planned also with different set ups where corresponding simulations have been carried out in the past (e.g. using atmospheric ion funnels [10]). Should the experimental results confirm the simulations, it becomes evident that atmospheric IMS coupled via an intermediate stage to vacuum IMS is, although offering striking simplicity in its set up, coming at the price of IMS resolving power. In order to get the ions into the MS, the IMS itself or its simplicity has to be sacrificed. Finally, this paper has shown how helpful such simulations can be when combining multi-physics approaches, such as electric fields, air currents calculated by CFD as well as ion motion under the influence of these fields and subject to diffusion effects for the effective development of e.g. gas monitors for environmental relevant applications.

\section{References}

1. G.A. Eiceman, Z. Karpas, Ion Mobility Spectrometry, CRC Press, Boca Raton, USA, (2005)

2. A.B. Kanu, P. Dwivedi, M. Tam, L. Matz, H.H. Hill, J. Mass Spectrom. 43, 1-22 (2008)

3. E. Hoffman, V. Stroobant, Mass Spectrometry Principles and Applications, John Wiley \& sons, Chichester, England (2007)

4. F. Gunzer, S. Zimmermann, W. Baether, Anal. Chem. 82, 3756 (2010)

5. W. Basther, S. Zimmermann, F. Gunzer, Int. J. Mobil. Spectrom. 13, 95 (2010)

6. W. Baether, S. Zimmermann, F. Gunzer, Sens. Act. B 171, 1238 (2012)

7. F. Gunzer, Int. J. Ion Mobil. Spectrom. 18, 41 (2015)

8. F. Gunzer, Analyst 140, 6379 (2015)

9. J.F. Wendt, Computational Fluid Dynamics - An Introduction, Springer Berlin Heidelberg, Germany (2009)

10. Q. Yu, Z. Diao, K. Ni, X. Qian, F. Tang, X. Wang, Rapid Commun. Mass Spectrom. 29, 1055 (2015) 